\title{
Recurrent Giant Sarcoma of the Anterior Abdominal Wall
}

\section{Horia Doran*}

"Dr I Cantacuzino" General Surgery Clinic, "Carol Davila” University of Medicine, Bucharest, Romania

\begin{abstract}
Abdominal wall sarcomas are rare tumors with a high recurrence rate. We present the case report of a 48-yearold female patient, who was admitted for an asymmetric, voluminous tumor in the lower abdomen, which had been rapidly increasing during the previous two months. The tumor was painless and fixed to the abdominal wall. Two other sarcomas had been resected from the same place, 2 years before-the former and 7 months before-the latter. The patient underwent a surgical procedure, consisting of a wide, tumor-free margin resection, which led to the removal of the rectus abdominis muscles under the umbilicus; abdominal wall reconstruction required a prosthetic mesh. Post-operative course did not include significant issues. Adjuvant radiotherapy was indicated, in order to decrease the risk of another recurrence.
\end{abstract}

\section{Introduction}

Sarcomas are a heterogeneous group of malignant neoplasms arising from mesenchymal cells. They account for less than $1 \%$ of adult solid malignant cancers. The vast majority are soft tissue sarcomas, while malignant bone tumors represent only about $10 \%$ of them [1]. In fact, sarcomas comprise over 50 different histological subtypes; they may arise in stem cell-like population (primitive neuroectodermal tumors) as well as from morphologically recognizable connective tissues [2]. Although the tumorigenesis of sarcoma is not well understood, the risk factors can be divided into environmental exposures, genetic susceptibility and an interaction between the two [1]. Considerable evidence supports a relationship between occupational chemicals, medicinal treatment, such as Thorotrast exposure, therapeutic irradiation and the development of soft tissue sarcoma [3].

The imagistic diagnosis is based on MRI, which provides information about the location and extent of these tumors. Definitive diagnosis obviously requires biopsy; the treatment of soft-tissue sarcomas is the surgical resection with a tumor-free margin [4]. The goal of curative surgical excision is en bloc excision of the tumor and any biopsy tracts with a negative margin of normal tissue of 1 to $2 \mathrm{~cm}$ [5]. The impact of positive margins on the outcome can be limited by the association of post-operative chemotherapy and radiotherapy [6]. Adjuvant radiotherapy is also indicated, because randomized trials have proved that it can be effective in decreasing local recurrence [7]. Mitotic rate, as well as tumor depth and size were reported to be significant predictors of development of recurrence and metastatic disease [8]. A recent study which analyzed 45,568 incident cases diagnosed between 1995 and 2002 in the 27 countries of the European Union stated that the five-year relative survival rate was $58 \%$ for soft tissue sarcomas and $62 \%$ for bone sarcomas [9]. In the USA, five-year survival rate has been estimated to approximately $90 \%$, for stage I sarcomas, gradually decreasing to $10 \%-20 \%$ for advanced stages [10].

\section{Case Report}

A 48-year-old female patient was admitted for an asymmetric, voluminous tumor in the lower abdomen (Figure 1). The patient had two children, no family history of neoplasia and did not smoke. However, she had been exposed to chemical agents (polyalcohols and isocyanates) in a shoe factory, where she had been working for twenty years. Her personal history also included two surgical procedures: the former had been performed two years before and had consisted of a surgical resection of a solid tumor, about 4 to $5 \mathrm{~cm}$ in size, located in the lower abdominal wall. It later proved to be a rabdomyosarcoma. As the histo-pathological examination had stated that the margins were tumor-free, no post-operative treatment had then been considered necessary.

The $2^{\text {nd }}$ tumor had developed after 15 months in the same place, inside the abdominal cavity, in front of the peritoneal layer (Figure 2) and also underwent a complete resection. It was obviously a recurrence, as the same histological type of rabdomyosarcoma was found; it was followed by post-operative radio-therapy for only two months. The patient did not complete the cure due to personal reasons.

A third, smaller tumor, of about 3 or $4 \mathrm{~cm}$, appeared approximately five to six weeks after the patient had interrupted the therapy. The tumor has been slowly developing during the first two months: afterwards, the growth rate suddenly increased, and the tumor reached its present size during the next two months, according to the patient's statement. No medical records were available regarding this evolution, as the patient had not addressed to a physician.

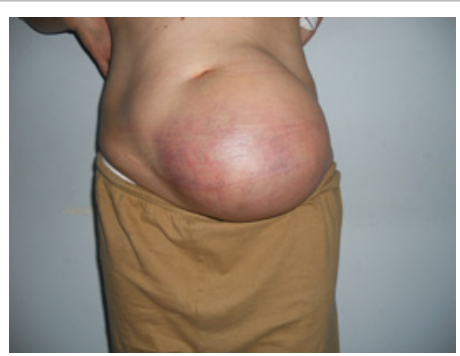

Figure 1: Clinical aspect: asymmetric, voluminous tumor in the lower abdomen.

*Corresponding author: Horia Doran, MD, PhD, Dr.l.Cantacuzino" Genera Surgery Clinic, "Carol Davila" University of Medicine, Sos.lancului nr.53 bloc102B scara C ap.101, 021719 Bucharest Romania, Tel: 004.0212504485; Fax: 004.0212101313; E-mail: doranh2003@yahoo.com

Received October 27, 2012; Accepted November 17, 2012; Published November 19, 2012

Citation: Doran H (2012) Recurrent Giant Sarcoma of the Anterior Abdomina Wall. J Cancer Sci Ther 4: 412-414. doi:10.4172/1948-5956.1000176

Copyright: (c) 2012 Doran H. This is an open-access article distributed under the terms of the Creative Commons Attribution License, which permits unrestricted use, distribution, and reproduction in any medium, provided the original author and source are credited. 
The clinical features which led to the diagnosis were: the size of more than $20 \mathrm{~cm}$, the fixation to the abdominal wall and the history of local surgical resection of two other sarcomas.

CT-scan showed a large non-homogenous tumor, whose origin was the rectus abdominis muscles and which extended in the left half of the abdominal wall, under the umbilical line (Figures 3 and 4).

The surgical procedure consisted of a wide resection of the tumor (Figure 5) and of the surrounding muscular layer. The complete resection of the rectus abdominis muscles under the umbilicus was necessary (Figure 6) in order to accomplish a tumor-free margin, which was stated by histological examination. The resection thus exceeded the macroscopic limits of the tumor by 4 to $5 \mathrm{~cm}$. Abdominal wall reconstruction required a prosthetic mesh (Figure 7), which was easily covered by the cutaneous flaps and their subcutaneous adipose tissue. Histological examination identified a rabdomyosarcoma, with the same features as the previously resected tumors.

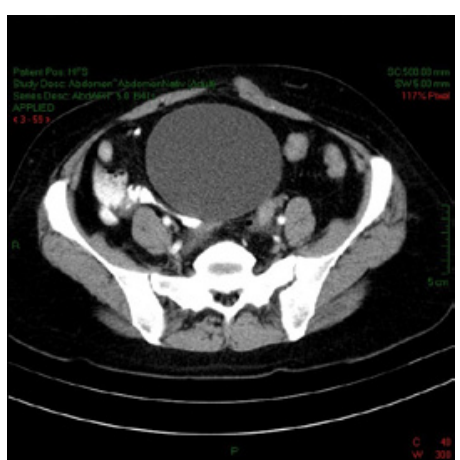

Figure 2: CT-scan: large tumor (rabdomyosarcoma) of the anterior abdominal wall, developed inside the abdomen.

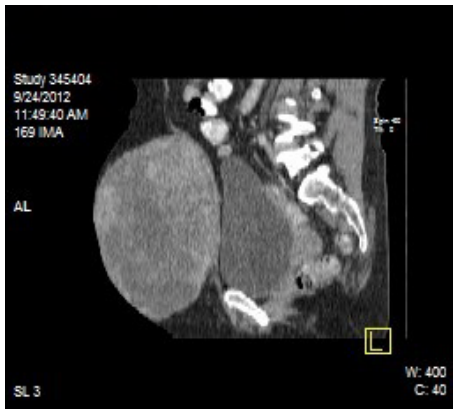

Figure 3: CT-scan: sagital section of the lower abdomen.

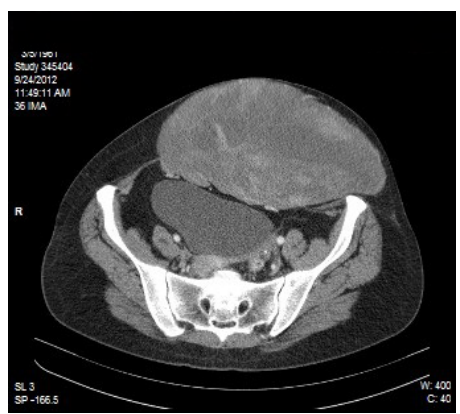

Figure 4: CT-scan: tranverse section of the lower abdomen.

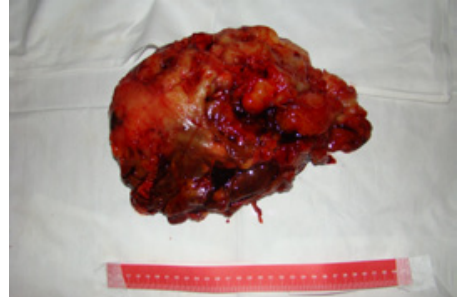

Figure 5: Aspect of the specimen

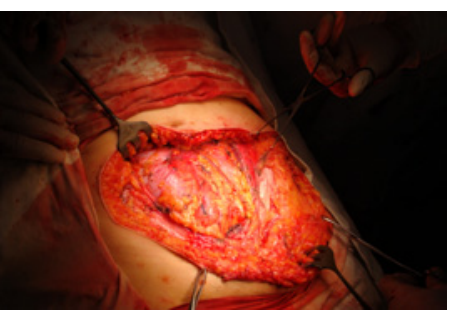

Figure 6: Intra-operative view: complete resection of the tumor and of the rectus abdominis muscles.

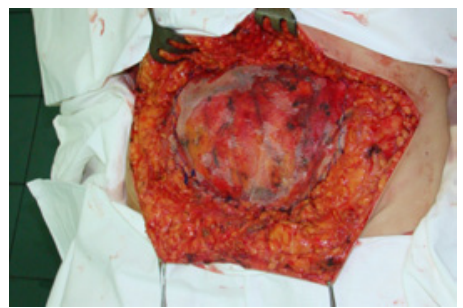

Figure 7: Intra-operative view: reconstruction of the abdominal wall with a prosthetic mesh.

Post-operative evolution did not include any significant issues. Post-operative radio-therapy was strongly indicated, due to the repeated recurrences of the tumor. The oncological treatment has now been in course for 4 months, with no clinical sign of local recurrence.

\section{Discussions}

Soft tissue sarcomas are quite rare neoplasma, which may occur in the extremities, as well as in the abdominal cavity and retroperitoneum, trunk, head and neck. More than 50 histologic subtypes have been described; the most frequent are liposarcoma, malignant fibrous histiocytoma and leiomyosarcoma [4]. Their etiology is yet to be agreed upon, although certain risk factors have been identified: genetic syndromes, radiation exposure, trauma and chemical agents. Our patient has been working in a shoe factory for twenty years, which led to her being exposed to occupational chemicals for a long period of time. Polyalcohols and isocyanates, which are two chemicals widely used in footwear manufacturing, can interfere with enzymatic activity and alter the structure of proteins, thus initiating the carcinogenetic process. It is reasonable to suppose that the occupational exposure of our patient might have caused the sarcoma, since no family history of tumors has been mentioned.

The personal history included two other tumors, which had developed and had been resected from the same anatomical site as the tumor in discussion. All these tumors had the same histological pattern, so there have obviously been two local recurrences of the 
initial lesion. As it usually occurs in this type of neoplasia, every new recurrence appeared after a shorter disease-free period and was larger than the previous one [8]. MRI with gadolinium is the elective method for imaging sarcomas, because it can evaluate the local anatomic extension, the involvement of contiguous structures and can also determine whether the lesion is a primary or a metastatic one [11].

The absence of radio-therapy after the former surgical procedures is another important issue that should be discussed. During the first operation, the histological statement of margin-free resection was accomplished. Still, as the oncological post-operative treatment is nowadays a widely indicated method $[4,6]$, it should not have been overlooked.

The present surgical procedure achieved a satisfactory wide resection of the tumor, which involved the surrounding muscular layer to a vast extent. Although the macroscopic limits of the tumor had already been exceeded by more than $3 \mathrm{~cm}$, the complete resection of the rectus abdominis muscles under the umbilicus was considered necessary, as previously shown in figure 6 . The aim of the procedure was to complete an oncological resection, which was deemed more important than the muscular layer shortage.

Reconstruction of the abdominal wall after a wide resection can be accomplished either by using plastic surgery techniques, such as myocutaneous innervated free flaps [12], or by prosthetic reconstruction techniques [13]. The latter was chosen due to the fact that it allows a more extensive resection; furthermore, it does not require laborious maneuveres involving blood vessels and nerves.

As previously stated, we considered post-operative radio-therapy a mandatory protocol for this patient, consistent with the data in medical literature. The current US National Comprehensive Cancer Network guidelines recommend radiation therapy in sarcomas for high-grade lesions, low-grade lesions larger than $5 \mathrm{~cm}$ (which is the case in this patient), or positive margins [14]. According to other studies, patients with reexcisions should routinely receive radio-therapy [10]. In a randomized trial conducted by the National Cancer Institute, surgery alone had a recurrence rate of $22 \%$ compared with a recurrence rate of less than $5 \%$ in patients receiving adjuvant radiotherapy [14].
The evolution of our patient will be decided by the individual response to the oncological treatment. Although she has been diseasefree for 4 months, the time-span is too limited even for a temporary conclusion.

\section{References}

1. Burningham Z, Hashibe M, Spector L, Schiffman JD (2012) The epidemiology of sarcoma. Clin Sarcoma Res 2: 14.

2. Thomas DM, Savage SA, Bond GL (2012) Hereditary and environmental epidemiology of sarcomas. Clin Sarcoma Res 2: 13.

3. Froehner M, Wirth MP (2001) Etiologic factors in soft tissue sarcomas Onkologie 24: 139-142.

4. Singer S, Brennan MF (2004) Soft Tissue Sarcomas, in Sabiston Textbook of Surgery $\left(17^{\text {th }}\right.$ edn), Philadelphia, PA: Elsevier $803-815$.

5. Cheng EY (2005) Surgical management of sarcomas. Hematol Oncol Clin North Am 19: 451-470.

6. Gronchi A, Verderio P, De Paoli A, Ferraro A, Tendero O, et al. (2012) Quality of surgery and neoadjuvant combined therapy in the ISG-GEIS trial on sof tissue sarcomas of limbs and trunk wall. Ann Oncol.

7. Steen S, Stephenson G (2008) Current treatment of soft tissue sarcoma. Proc (Bayl Univ Med Cent) 21: 392-396.

8. Radkowski CA, Dodd LG, Johnson JL, Harrelson JM, Brigman BE (2012) Leiomyosarcoma of the somatic soft tissues. J Surg Orthop Adv 21: 96-101.

9. Stiller CA, Trama A, Serraino D, Rossi S, Navarro C, et al. (2012) Descriptive epidemiology of sarcomas in Europe: Report from the RARECARE project. Eur J Cancer.

10. Nedea EA, DeLaney TF (2006) Sarcoma and skin radiation oncology. Hematol Oncol Clin North Am 20: 401-429.

11. Kurland BF, Gerstner ER, Mountz JM, Schwartz LH, Ryan CW, et al. (2012) Promise Promise and pitfalls of quantitative imaging in oncology clinical trials. Magn Reson Imaging 30: 1301-1312.

12. Grinsell D, Di Bella C, Choong PF (2012) Functional reconstruction of sarcoma defects utilising innervated free flaps. Sarcoma 2012: 315190.

13. Donati M, Gandolfo L, Brancato G, Caglià P, Cavallaro G, D'Addea I, et al (2003) Surgical tactics in abdominal wall neoplasia. Tumori 89: 61S-62S.

14. Kaushal A, Citrin D (2008) The role of radiation therapy in the management of sarcomas. Surg Clin North Am 88: 629-646. 